\title{
Recruiting care homes to a randomised controlled trial
}

\author{
Alison Ellwood ${ }^{1 *}$, Jennifer Airlie ${ }^{1,2}$, Robert Cicero ${ }^{3}$, Bonnie Cundill ${ }^{3}$, David R Ellard ${ }^{4}$, Amanda Farrin $^{3}$, \\ Mary Godfrey ${ }^{1,5}$, Liz Graham¹, John Green ${ }^{1}$, Vicki McLellan, Najma Siddiqi ${ }^{6}$, Anne Forster ${ }^{1,5}$ \\ and On behalf of the REACH Programme Team
}

\begin{abstract}
Background: There are more than a quarter of a million individuals aged $\geq 65$ years who are resident in care homes in England and Wales. Care home residents have high levels of cognitive impairment, physical disability, multimorbidity and polypharmacy. Research is needed to ensure there are robust, evidence-based interventions to improve the quality of life of this frail group. However, there is a paucity of research studies in this area. Recruiting care homes and their residents to research is challenging.

A feasibility, cluster randomised controlled trial was undertaken as part of a research programme to identify ways to develop and test methods to enhance the physical activity of care home residents. This paper describes two methods of recruiting care homes to the trial and draws out learning to inform future studies.
\end{abstract}

Methods: Eligible care homes met the following criteria: they were within a defined geographical area in the north of England; provided residential care for adults $\geq 65$ years of age; had not previously been involved in the research programme; were not taking part in a conflicting study; were not recorded on the Care Quality Commission website as 'inadequate' or 'requiring improvements' in any area; and had $\geq 10$ beds. Care homes were identified by a 'systematic approach' using the Care Quality Commission website database of care homes or a 'targeted approach' via a network of research-ready care homes. A standardised method was used to recruit care homes including eligibility screening; invitation letters; telephone contact; visits; formal letter of agreement.

Results: In the systematic approach, 377 care homes were screened, 230 (61\%) were initially eligible and invited to participate, 11 were recruited (recruitment rate (RR) 4.8\%). In the targeted approach, 15 care homes were invited to participate, two were recruited (RR 13.3\%). Overall, 245 care homes were approached and 13 recruited (RR 5.3\%). A variety of care homes were recruited to the trial in terms of size, location, ownership and care provision.

Conclusions: Systematic recruitment of care homes to the study was time-consuming and resource-heavy but led to a variety of care homes being recruited. The targeted approach led to a higher recruitment rate.

Trial registration: ISRCTN registry, ISRCTN16076575. Registered on 25 June 2015.

Keywords: Homes for the aged, Aged, Aged, 80 and over, Randomised controlled trials as topic, Research subjects, Vulnerable populations, United Kingdom, Recruitment, Care homes

\footnotetext{
* Correspondence: alison.ellwood@bthft.nhs.uk

${ }^{1}$ Academic Unit of Elderly Care and Rehabilitation, Bradford Institute for

Health Research, Bradford Teaching Hospitals NHS Foundation Trust, Temple

Bank House, Bradford Royal Infirmary, Duckworth Lane, Bradford, West

Yorkshire BD9 6RJ, UK

Full list of author information is available at the end of the article
}

(c) The Author(s). 2018 Open Access This article is distributed under the terms of the Creative Commons Attribution 4.0 International License (http://creativecommons.org/licenses/by/4.0/), which permits unrestricted use, distribution, and reproduction in any medium, provided you give appropriate credit to the original author(s) and the source, provide a link to the Creative Commons license, and indicate if changes were made. The Creative Commons Public Domain Dedication waiver (http://creativecommons.org/publicdomain/zero/1.0/) applies to the data made available in this article, unless otherwise stated. 


\section{Background}

Between 2005 and 2015, the UK population aged 65 years and over increased by $21 \%$, and the population aged 85 years and over increased by 31\% [1]. In 2011, 291,000 individuals aged 65 years and over lived in care homes (CHs) in England and Wales [2]. CHs 'offer accommodation and personal care for people who may not be able to live independently. Some homes also offer care from qualified nurses or specialise in caring for particular groups' [3], for example, people with dementia. With projected demographic changes the number of people living in $\mathrm{CHs}$ is likely to increase in the future [4]. $\mathrm{CH}$ residents are among the frailest of the population, distinguishable from community-dwelling older adults of the same age because of their dependency on others, cognitive impairment, multimorbidity and polypharmacy [5]. Accordingly, research specifically focused on the $\mathrm{CH}$ setting is necessary to try and address the challenges of health care for these vulnerable people and ensure robust, evidence-based service improvements are developed and implemented [6].

However, the $\mathrm{CH}$ setting has been largely neglected in research, particularly in clinical trials [7]. This could be partially attributed to the complexities and increased costs associated with recruiting vulnerable people, particularly those with cognitive impairment, to studies [8]. However, recruitment of vulnerable older adults to research has reported low decline rates, suggesting their willingness to be involved when given the opportunity $[9,10]$. Whilst this is promising, recruiting $\mathrm{CH}$ residents to research presents particular challenges, including the need first to recruit $\mathrm{CHs}$.

Recruiting $\mathrm{CHs}$ to research is also challenging. Access to $\mathrm{CHs}$ to conduct research requires the consent of managers and providers prior to approaching residents [11]. Unlike the National Health Service (NHS), the majority of $\mathrm{CH}$ providers are independent businesses offering a service for financial gain. $\mathrm{CHs}$ are often subject to scrutiny by the media, experience high staff turnover and must contend with pressurised situations, so allowing research teams to access their businesses may not be prioritised. Additionally, $\mathrm{CH}$ employees are often unfamiliar with research processes (e.g. randomisation, 'blinding' of researchers). Thus, studies undertaken in $\mathrm{CHs}$ may require greater researcher input when compared to similar work in hospitals or in the community [12]. It is likely that all these factors contribute to the paucity of involvement from the sector. In practice, this means that many $\mathrm{CH}$ studies are open to bias, as they are undertaken within homes preselected either by organisations [13] or through previous research involvement [10]. To address the problem of under-representation of $\mathrm{CHs}$ and their residents in high-quality studies, $\mathrm{CH}$ research networks (for example, Enabling Research in Care
Homes - ENRICH) have been established [14, 15]. However, this may also result in potential bias by recruiting homes from a select group rather than offering involvement to a wider pool.

The Research Exploring Physical Activity in Care Homes (REACH) research programme sought to identify ways to develop and test methods to enhance the physical activity of $\mathrm{CH}$ residents. The final workstream of the programme was a feasibility, cluster randomised trial (CRT) of the REACH intervention plus usual care compared to usual care alone in preparation for a future definitive large-scale trial [16]. Data collection was at baseline and at 3, 6 and 9 months and required $\mathrm{CH}$ staff to collect data and to support residents with completion of outcome measures, including the wearing of an accelerometer on an elasticated belt around the waist and logging of time worn. Additionally, the intervention required a range of staff in the $\mathrm{CH}$ to be released to attend a series of three workshops and then to work with all the $\mathrm{CH}$ staff as a team to develop ways to encourage residents to improve their levels of movement. A systematic, rigorous and robust methodology was sought to give $\mathrm{CHs}$ within a defined geographical area the opportunity to participate in the trial, thereby decreasing selection bias. This paper describes the methods of recruiting $\mathrm{CHs}$ to the $\mathrm{REACH}$ feasibility $\mathrm{CRT}$ and the complexities encountered when recruiting $\mathrm{CHs}$ to involvement in a CRT. This process enabled a comparison to be made between two methods of recruiting $\mathrm{CHs}$ to the study: the systematic recruiting of $\mathrm{CHs}$ in a defined geographical area and recruitment via the local ENRICH network.

\section{Methods}

$\mathrm{CHs}$ were eligible for the study if they were within a defined geographical area in the north of England, provided residential care (with or without additional specialist nursing and/or dementia care) for adults 65 years of age and over, had not been involved in previous $\mathrm{REACH}$ workstreams, were not taking part in a conflicting study or were not recorded on the Care Quality Commission (CQC) [3] website as 'inadequate' or 'requiring improvements in any area', as it was felt that the additional workload of a programme of enhanced care would have proved burdensome to homes addressing these concerns. In addition, for trial efficiency, it was agreed only to contact $\mathrm{CHs}$ with at least 10 beds to ensure that sufficient residents would be recruited to the study. In large multisite or multifloor establishments, one or two units within the home were eligible to be selected to participate as one home. The chosen units were identified by the home manager in discussion with the researcher. 
The standardised method used by the research team for $\mathrm{CH}$ recruitment to the trial had four stages: 1 . $\mathrm{CHs}$ were screened for eligibility and invitation letters were sent to eligible $\mathrm{CHs} ; 2$. CHs were contacted by telephone; 3 . initial visits to the $\mathrm{CH}$ were undertaken; 4 . interested CHs were given a letter of agreement to sign. There were three waves of recruitment using $\mathrm{CHs}$ identified through the CQC (labelled 'systematic' recruitment) and one using the ENRICH network (labelled 'targeted' recruitment). The plan was to recruit and randomise two $\mathrm{CHs}$ per month over a 6-month period.

\section{Systematic recruitment: first wave}

\section{Stage 1: screening for eligibility and invitation letters}

In June 2015, CHs in West Yorkshire were identified using the publicly available care directory on the CQC website. This dataset was filtered to include only $\mathrm{CHs}$ providing residential care (with or without additional specialist nursing and/or dementia care) from the five local authority areas (population 2,226,058 in 2011 [17]), with 10 or more beds and which were categorised under the 'older people' service user band. All CHs identified were individually screened using the eligibility criteria. Eligible CHs were then sent an information pack by post inviting them to consider participation in the $\mathrm{REACH}$ feasibility trial. The pack included an introductory letter, information sheet about the study and a reply slip with which to register interest or to decline participation.

\section{Stage 2: telephone contact}

On receipt of reply slips registering interest in the study, researchers re-checked that the $\mathrm{CHs}$ were not ineligible (i.e. they were exclusively a nursing home or provided care for younger people) and their current status on the $\mathrm{CQC}$ website. The $\mathrm{CHs}$ were then telephoned to confirm their eligibility, to discuss the likely resource requirements for participating ( $\mathrm{CH}$ staff assisting with data collection and, depending on randomisation, possible involvement in the intervention) and to confirm they had no current involvement in any other conflicting research trials or studies.

Attempts were made to telephone $\mathrm{CH}$ managers who did not respond to the initial letter about the study after first having checked on the CQC website that their status had not changed and that they remained open and provided residential care for older people. Eligibility criteria were confirmed when contact was made with these $\mathrm{CHs}$. Those expressing interest were visited as described in the following section.

\section{Stage 3: initial visits}

Meetings were arranged with the managers of $\mathrm{CHs}$ still interested in being involved following the initial telephone conversation. Further details about the study, time commitments, staff roles and trial processes were provided at this meeting, and any queries $\mathrm{CH}$ managers had were answered.

\section{Stage 4: letter of agreement}

$\mathrm{CHs}$ agreeing to participate were asked to sign a formal letter of agreement between both the $\mathrm{CH}$ manager and owner and the research sponsor (Bradford Teaching Hospitals NHS Foundation Trust) to enable them to participate in the study. The letter of agreement set out the duties and responsibilities of both parties, including agreement to allow access to the $\mathrm{CH}$ for the $\mathrm{REACH}$ research team for the purposes of data collection over four specified periods and agreement to make a modest payment of $£ 600$ to the $\mathrm{CH}$ for continued involvement in the study.

Following the poor response rate to the first wave of recruitment outlined above, two further recruitment waves via the CQC database were made. These are outlined in the following text.

\section{Systematic recruitment: second wave}

In January 2016, CHs initially deemed as ineligible due to their CQC status were re-screened. CHs now re-rated as 'good' or requiring improvements in only one area of the CQC report were invited to participate, using the process outlined in the first wave as previously described.

\section{Systematic recruitment: third wave}

In February 2016, the initial screening area was widened, and residential $\mathrm{CHs}$ from specific areas of North Yorkshire were identified (using the same CQC directory from stage 1), screened and approached using the same recruitment process as outlined previously for the first wave. As with the second wave, homes were included if they required improvements in only one area. ENRICH homes already approached were excluded (see the following section).

\section{Targeted recruitment through the ENRICH network}

In January 2016, the Yorkshire Enabling Research in Care Homes (ENRICH) network assisted with contacting $\mathrm{CHs}$ in their network within specified areas of North Yorkshire. Invitations were emailed to each $\mathrm{CH}$ with details on how to express interest in participating in the study. Details of $\mathrm{CHs}$ expressing interest in the study were passed on to researchers to establish eligibility and to contact and arrange visits as outlined previously for the first wave.

Researchers kept contemporaneous notes of all visits and contacts with $\mathrm{CHs}$. 


\section{Results}

$\mathrm{CH}$ screening commenced in June 2015, the final $\mathrm{CH}$ letter of agreement was signed in July 2016 and the final $\mathrm{CH}$ was randomised in September 2016. The recruitment target of $12 \mathrm{CHs}$ was achieved in 16 months. This required the equivalent of approximately one full-time researcher. Figures 1 and 2 are Consolidated Standards of Reporting Trials (CONSORT) diagrams showing respectively the flow of $\mathrm{CHs}$ through the recruitment processes for systematic recruitment via the CQC databases (the first to third waves) and targeted recruitment via the ENRICH network.

\section{Stage 1: CH screening and invitation letter}

\section{Systematic recruitment: first to third waves}

Three hundred and seventy-seven $\mathrm{CHs}$ were screened, and 147 (39.0\%) were excluded. Two hundred and thirty $\mathrm{CHs}$ were invited to participate in the study. Of the 36
$\mathrm{CHs}$ (15.7\%) responding to the invitation letter, 22 (9.6\%) expressed interest in the study: 61\% of those responding. These 22 interested $\mathrm{CHs}$, and the $194 \mathrm{CHs}$ not responding to the invitation letter, were re-screened before an attempt was made to contact them via telephone. Fifty-two were found to be ineligible despite being initially eligible at the first screening, due to changes in CQC status, closures and ineligible service provision.

\section{Targeted recruitment through the ENRICH network}

Fifteen $\mathrm{CHs}$ in the ENRICH network were emailed by a senior community research nurse from the Yorkshire and Humber Clinical Research Network, based upon location but not screened for eligibility, and invited to participate in the study. Six $\mathrm{CHs}(40 \%)$ responded to the invitation email, all expressing interest in the study. A further nine $\mathrm{CHs}$ not responding to the invitation email

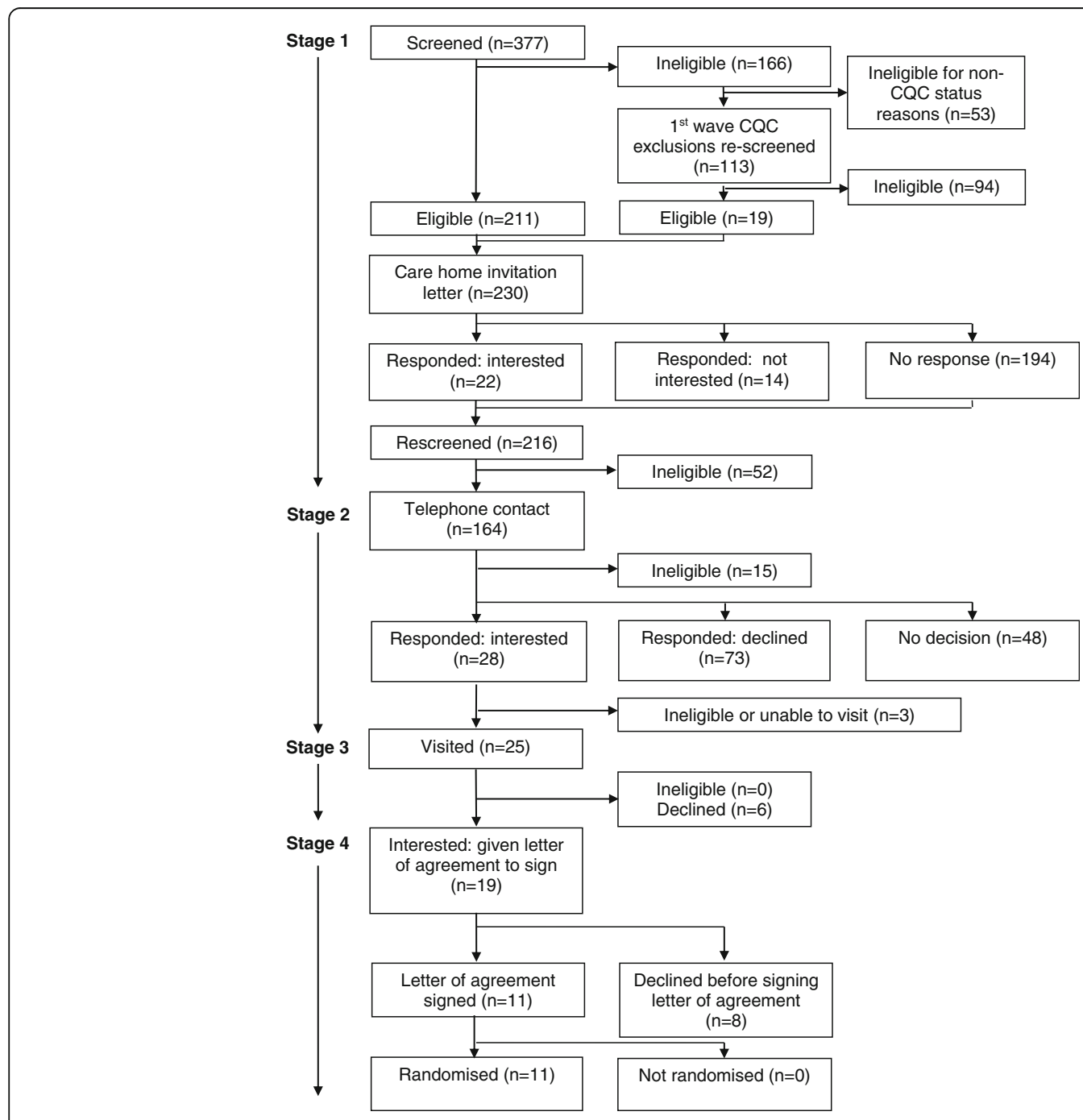

Fig. 1 Consort diagram: care home recruitment flow chart for first to third waves ('systematic' recruitment) 


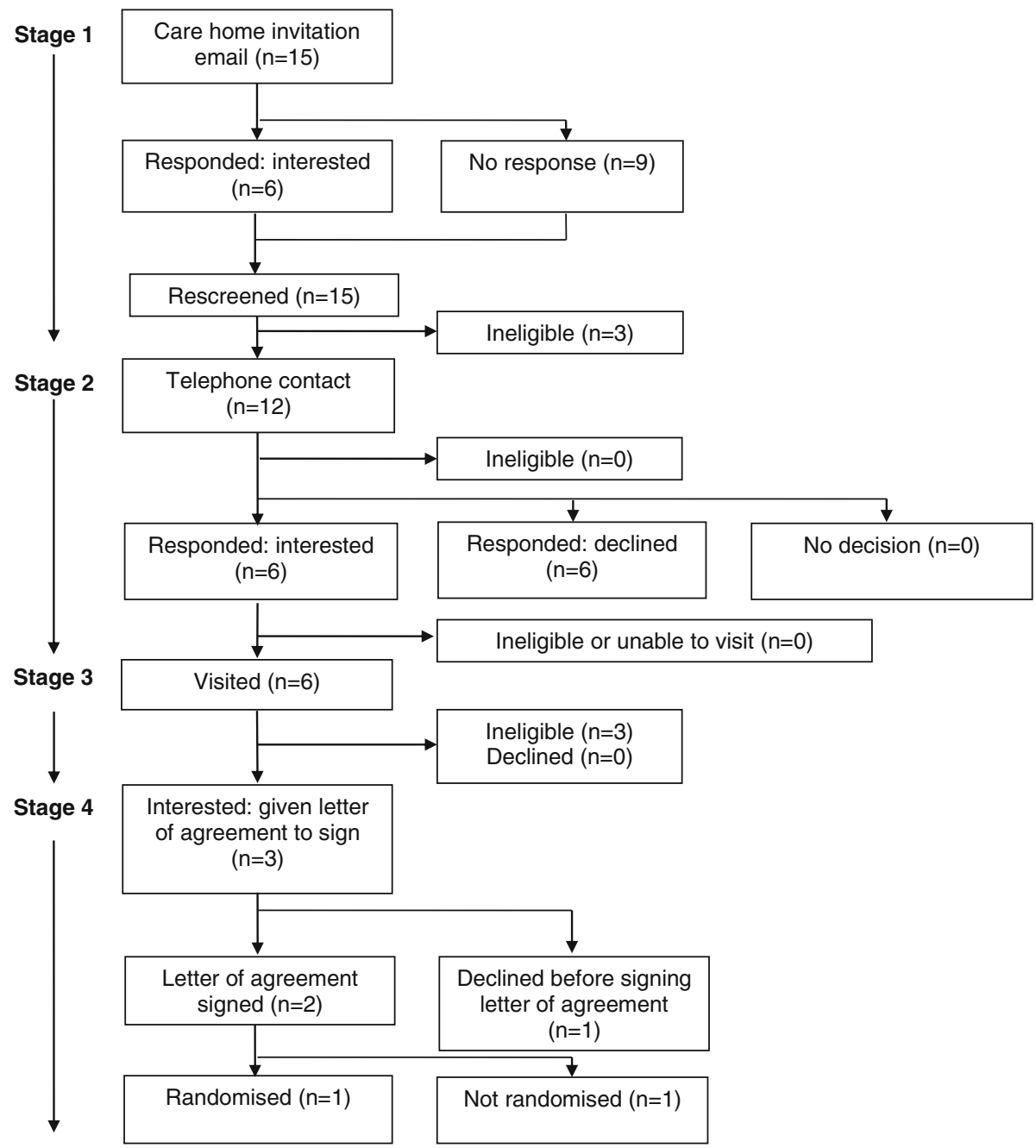

Fig. 2 Consort diagram: care home recruitment flow chart for 'targeted' recruitment via the ENRICH network

were re-screened before an attempt was made to contact them via telephone. Three were found to be ineligible, due to changes in CQC status and ineligible service provision.

\section{Stage 2: $\mathrm{CH}$ telephone contact}

Telephone contact was made with 164 eligible $\mathrm{CHs}$ in the first to third waves and 12 of the ENRICH network $\mathrm{CHs}$. Of these, 15 (all systematic approach $\mathrm{CHs}$ ) were deemed ineligible, 34 (28 systematic and 6 targeted) expressed (or maintained) interest and 79 (73 systematic and 6 targeted) declined, typically citing 'busyness' or lack of interest in research as the reason (Table 1). The remaining $48 \mathrm{CHs}$ (all systematic) had initially expressed interest and requested further information via email, post or follow-up calls. However, this interest was not maintained despite the continued efforts of the research team. Three of the 34 interested $\mathrm{CHs}$ contacted by telephone expressed interest, but a visit could not be arranged or they were later found to be ineligible.

\section{Stage 3: $\mathrm{CH}$ meetings}

The research team visited 31 of the 34 interested $\mathrm{CHs}$ (25 systematic and 6 targeted) 47 times to discuss the study: systematic (median (interquartile range [IQR]; range)) visits: $2(1-2 ; 1-3)$; targeted: 1 visit each $\mathrm{CH}$. Of the $31 \mathrm{CHs}$ visited, three were found to be ineligible (all targeted ENRICH CHs), and six CHs declined participation (all systematic sites). The remaining $22 \mathrm{CHs}$ (19 systematic and 3 targeted) were given a letter of agreement to sign.

\section{Stage 4: letter of agreement}

A letter of agreement was provided to $22 \mathrm{CHs}$ (19 systematic and 3 targeted). Despite repeated contact from the research team, nine did not sign the letter of agreement (8 systematic $\mathrm{CHs;} 1$ targeted $\mathrm{CH}$ ). Five managers, including the targeted site, declined due to difficulties engaging their head offices in the project, and four experienced a change in circumstances affecting their 
Table 1 Reasons for non-participation of care homes at telephone contact stage

\begin{tabular}{llll}
\hline Reason & Systematic $(n=73)$ & Targeted $(n=6)$ & Total $(n=79)$ \\
\hline Too busy & $20(27.4 \%)$ & $0(0 \%)$ & $20(25.3 \%)$ \\
Unable to contact & $14(19.2 \%)$ & $0(0 \%)$ & $14(17.7 \%)$ \\
Not interested in research & $11(15.1 \%)$ & $0(0 \%)$ & $11(13.9 \%)$ \\
Unstable management or home & $11(15.1 \%)$ & $0(0 \%)$ & $11(13.9 \%)$ \\
$\begin{array}{l}\text { Participation in research initiated } \\
\text { only through head office }\end{array}$ & $4(5.5 \%)$ & $0(0 \%)$ & $4(5.1 \%)$ \\
Disapproves of research & $1(1.4 \%)$ & $0(0 \%)$ & $1(1.3 \%)$ \\
No reason given & $10(13.7 \%)$ & $6(100.0 \%)$ & $10(12.7 \%)$ \\
Missing data & $2(2.7 \%)$ & $8(10.1 \%)$
\end{tabular}

capacity fully to engage with the project. Thirteen $\mathrm{CHs}$ signed the letter of agreement, and $12 \mathrm{CHs}$ were subsequently randomised; one of these was a targeted $\mathrm{CH}$. The remaining $\mathrm{CH}$ (a targeted home) was not randomised because the required sample size had then been reached.

The overall recruitment rate was 5.3\% (13 CHs recruited from 245 approached by post or email). The recruitment rate for the systematic approach was lower than that of the targeted approach: $4.8 \%$ (11 CHs recruited from 230 approached) versus 13.3\% (2 CHs recruited from 15 approached) respectively.

The average time taken to recruit the $\mathrm{CHs}$ (from initial visit to gaining access to screen residents for eligibility) varied. In the systematic approach $\mathrm{CHs}$, this ranged from 7 to 134 days (median (IQR) 56 (32.25-92.75) days); of the two targeted approach $\mathrm{CHs}$ recruited, only one was randomised, and the time between initial visit and access to residents for data collection was 97 days. The time taken to recruit the independently owned $\mathrm{CHs}$ was less than for the $\mathrm{CHs}$ run by larger organisations (median (IQR) days: 48 (17-59) versus 80 (54-131) respectively).

A variety of $\mathrm{CHs}$ were randomised to the $\mathrm{REACH}$ trial in terms of size, location, ownership and care provision (Table 2). Units from three $\mathrm{CHs}$ were recruited rather than the whole $\mathrm{CH}$. Nine $\mathrm{CHs}$ (all systematic) were classified as small/medium ( $\leq 40$ beds) [15], and four as large (> 40 beds). The median (IQR; range) of beds in the $\mathrm{CHs}$ or units was 29 (18-37.5; 12-64) beds. Six CHs were located in suburban locations, four in semi-rural locations, two in urban locations and one in a rural area (the $\mathrm{CH}$ recruited through the ENRICH network). Five $\mathrm{CHs}$ were independently owned, three were part of not-for-profit organisations, four were part of a 'chain' and one was owned by a local authority. In addition to residential care, one $\mathrm{CH}$ provided nursing care and one $\mathrm{CH}$ provided respite care. The three $\mathrm{CHs}$ where units were involved additionally provided specialist residential dementia care.

\section{Discussion}

A systematic, standardised and rigorous procedure was adopted to recruit $\mathrm{CHs}$ to a feasibility CRT in a defined geographical area to assess the suitability of this method of recruitment for a large-scale definitive trial. This was supplemented by targeted recruitment using a similar standardised method. A range of $\mathrm{CHs}$ were recruited in terms of size, location, ownership and provision (Table 2). However, recruitment proved to be time-consuming and resource-heavy: it took approximately 16 months from the start of the initial screening to randomising the final $\mathrm{CH}$ and approximately the equivalent of one full-time researcher recruiting $\mathrm{CHs}$ via either approach. In order to recruit the target of $12 \mathrm{CHs}, 245 \mathrm{CHs}$ were approached by post or email. The recruitment rates of $5.3 \%$ overall and $4.8 \%$ for the systematic approach and $13.3 \%$ for the targeted approach are far lower than others reported (27-73\%) [14]. However, many studies do not explicitly describe how $\mathrm{CHs}$ were recruited. It may be they sourced 'research-ready' $\mathrm{CHs}$ and did not approach all eligible $\mathrm{CHs}$ within a geographical area.

There was a poor response by $\mathrm{CHs}(16 \%)$ to the first invitation letter (first to third waves of systematic recruitment). Many $\mathrm{CHs}$ subsequently explained that they did not recall seeing the information or had been too busy to respond to it. Targeted recruitment proved more successful: out of 15 ENRICH network $\mathrm{CHs}$ emailed, six (40\%) responded to the invitation. However, given that $\mathrm{CHs}$ in the ENRICH network are 'research-ready', this response was disappointing.

Concurrent changes to the CQC inspection process led to many $\mathrm{CHs}$ requiring some improvements. It was noted that minor improvements required to medication administration procedures and deprivation of liberty safeguarding documentation were frequently recorded on $\mathrm{CQC}$ reports. On review, these were not deemed by the research team, which included colleagues with experience in $\mathrm{CH}$ work, as likely to affect their capacity to undertake the REACH research project or intervention. $\mathrm{CHs}$ requiring improvements in one area are still rated 
Table 2 Characteristics of recruited care homes

\begin{tabular}{|c|c|c|c|}
\hline & Systematic $(n=11)$ & Targeted $(n=2)$ & Total $(n=13)$ \\
\hline \multicolumn{4}{|l|}{ Size } \\
\hline Small/medium & $9(81.8 \%)$ & $0(0 \%)$ & $9(69.2 \%)$ \\
\hline Large & $2(18.2 \%)$ & $2(100.0 \%)$ & $4(30.8 \%)$ \\
\hline \multicolumn{4}{|l|}{ Recruited component } \\
\hline Care home & $9(81.8 \%)$ & $1(50.0 \%)$ & $10(76.9 \%)$ \\
\hline Unit & $2(18.2 \%)$ & $1(50.0 \%)$ & $3(23.1 \%)$ \\
\hline \multicolumn{4}{|c|}{ Number of beds in care home or unit } \\
\hline Mean (SD) & $28(10.26)$ & $41(32.53)$ & $30(14.13)$ \\
\hline Median (IQR) & $29(18-35)$ & 41 & $29(18-37.5)$ \\
\hline Range & $12-44$ & $18-64$ & $12-64$ \\
\hline \multicolumn{4}{|l|}{ Location } \\
\hline Urban & $2(18.2 \%)$ & $0(0 \%)$ & $2(15.4 \%)$ \\
\hline Suburban & $6(54.5 \%)$ & $0(0 \%)$ & $6(46.2 \%)$ \\
\hline Semi-rural & $3(27.3 \%)$ & $1(50.0 \%)$ & $4(30.8 \%)$ \\
\hline Rural & $0(0 \%)$ & $1(50.0 \%)$ & $1(7.7 \%)$ \\
\hline \multicolumn{4}{|l|}{ Ownership } \\
\hline Independent & $5(45.5 \%)$ & $0(0 \%)$ & $5(38.5 \%)$ \\
\hline Chain & $2(18.2 \%)$ & $2(100.0 \%)$ & $4(30.8 \%)$ \\
\hline Not-for-profit & $3(27.3 \%)$ & $0(0 \%)$ & $3(23.1 \%)$ \\
\hline Local authority & $1(9.1 \%)$ & $0(0 \%)$ & $1(7.7 \%)$ \\
\hline \multicolumn{4}{|c|}{ Provision of care in care home or unit } \\
\hline Residential & $8(72.7 \%)$ & $0(0 \%)$ & $8(61.5 \%)$ \\
\hline Residential, dementia & $2(18.2 \%)$ & $1(50 \%)$ & $3(23.1 \%)$ \\
\hline Residential, nursing & $1(9.1 \%)$ & $0(0 \%)$ & $1(7.7 \%)$ \\
\hline Residential, dementia, respite & $0(0 \%)$ & $1(50 \%)$ & $1(7.7 \%)$ \\
\hline
\end{tabular}

$S D$ standard deviation, $I R Q$ interquartile range

as 'good' for the purposes of CQC auditing. The decision was therefore made to relax the initial inclusion criterion relating to CQC status from 'no improvements needed' to 'one improvement needed' in a second and a third wave of screening.

Given the continual state of flux of the $\mathrm{CH}$ sector, repeated checking of $\mathrm{CH}$ eligibility was deemed necessary throughout the recruitment process. This time-consuming but essential process was repeated each time a $\mathrm{CH}$ was telephoned: of the $\mathrm{CHs}$ re-screened prior to telephone contact, 55 (24\%) were found to be ineligible or excluded despite being initially eligible at the first screening. This also has implications for the conduct of trials, particularly those that are of any significant duration, as homes that were initially eligible may subsequently become ineligible in a short space of time.

Maintaining the interest of $\mathrm{CHs}$ required frequent contact from the researchers. The changeable and distinctive $\mathrm{CH}$ settings required flexibility, sensitivity and extensive planning from the researcher, who was experienced in $\mathrm{CH}$ work, including the timing of visits, adapting to last- minute cancellations and an awareness of the low priority given to research. Provision of $\mathrm{CH}$ information packs was helpful in the meetings with $\mathrm{CH}$ managers, many of whom had had little experience with such research.

The burdens of data collection and the requirements of the intervention on their staff were important considerations for managers in deciding whether to participate or not. For some, the staff time necessary to support data collection by researchers was reckoned to be too great. A number of managers also remarked they would participate in studies requiring less of their time, e.g. completion of questionnaires.

Managers also balanced the requirements of the intervention on their staff with what they perceived as free 'training'. Indeed, some were particularly keen to receive the 'training' despite being repeatedly informed of the random allocation to intervention or usual care. A number of $\mathrm{CH}$ managers may have declined because the intervention and associated training did not accord with mandatory training or fit with existing practice. 
Obtaining signatures from both the $\mathrm{CH}$ manager and owner on a letter of agreement, a formal requirement for $\mathrm{CH}$ participation, was usually straightforward in independently run private businesses but, despite $\mathrm{CH}$ managers being keen to be involved in research [11], was often problematic and time-consuming in larger $\mathrm{CH}$ organisations. Indeed, in some cases, these delays caused eventual withdrawal of interest from homes [14]. Despite being part of a research network, there was little evidence that this process was more straightforward or expedited in the targeted $\mathrm{CHs}$, where obtaining head office signatures encountered the same delays as for the systematic $\mathrm{CHs}$. A subsequent reduction in the length and complexity of the letter of agreement appeared to expedite the sign-off in the later stages of $\mathrm{CH}$ recruitment. The position large corporations took regarding research also affected uptake. A number of $\mathrm{CHs}$ indicated that they would only take on research projects signposted to them by their head office, or in some cases they had research programmes of their own.

To sustain $\mathrm{CHs}^{\prime}$ interest in the study, protracted and repeated contacts (face-to-face meetings and telephone calls) were often necessary between the initial expression of interest and researchers gaining access to the $\mathrm{CH}$ to screen residents. More visits were undertaken to the systematic approach $\mathrm{CHs}$ than to the targeted approach $\mathrm{CHs}$. This was more a reflection of their location further away from the researcher base rather than their being part of the ENRICH network. Interest was most difficult to sustain in the period after the letter of agreement had been provided to $\mathrm{CHs}$ but had not been signed by the owner. This was less difficult, however, in independent $\mathrm{CHs}$, where the owner was usually more readily available.

Intervention studies in $\mathrm{CHs}$, especially those requiring input from the whole home (staff, residents and management) are uncommon. Previous research has typically involved collection of data from databases and records or from resident or staff interviews or observational work $[18,19]$, which was consonant with the views expressed by some $\mathrm{CH}$ managers. Development of the ENRICH network reflects both the need of studies such as REACH and the difficulty in attracting $\mathrm{CHs}$ to involvement in research. Although development of such networks is largely positive, their use may limit access to other $\mathrm{CHs}$ for the purpose of research, leading to a biased sample. Utilisation of the ENRICH network for this study yielded greater interest than mailshotting $\mathrm{CHs}$; however, only two homes of the 15 approached signed the letter of agreement (13.3\%). This suggests that additional work could be done by the ENRICH network to improve CH staff members' understanding of the variety of research projects in which they may be able to engage.

Previous REACH workstreams and other studies utilised a more ad hoc method of recruiting $\mathrm{CHs}$ via forums, previous contact, 'snowballing' or approaching the head offices of large chains $[20,21]$. This may have required less researcher time. However, the aim of the present study was to establish a systematic, rigorous and robust methodology to recruit a representative and unbiased sample of $\mathrm{CHs}$, capable of replication in a future large-scale trial. Although this methodology reduces bias, recruited $\mathrm{CHs}$ were still self-selecting and subject to eligibility criteria, which limited involvement. This may have an impact on generalisability of findings if only interested and 'good' homes are involved. Involving $\mathrm{CHs}$, which are usually private businesses and run for financial gain, will carry a risk of bias towards those homes which practise an open-door policy and welcome involvement from external parties. Using CQC status as an eligibility criterion excluded access to the study for many $\mathrm{CHs}$ in the geographical area. Whilst the CQC status of a number of $\mathrm{CHs}$ changed during involvement with $\mathrm{REACH}$, engagement by managers and staff with the requirements of the research in these homes was largely consistent over time, perhaps calling into question the purpose of restricting approach only to homes with a 'good' rating. It is possible that $\mathrm{CHs}$ may utilise involvement in research as evidence of steps taken to improve service provision. Potential change of status meant that this eligibility criterion may therefore be both unstable and an unreliable means of selecting $\mathrm{CHs}$ to approach.

The recruitment method utilised two sources of potential CHs: a systematic approach via a comprehensive database of $\mathrm{CHs}$ accessed through the $\mathrm{CQC}$ website, and a targeted approach via a network of research-ready care homes (ENRICH). Although only two $\mathrm{CHs}$ were recruited via the ENRICH network, the recruitment rate was higher than that obtained via the systematic approach using the CQC database (13.3\% versus $4.8 \%$ ), which suggests a less labour-intensive method of $\mathrm{CH}$ recruitment. However, as only two $\mathrm{CHs}$ were recruited via the ENRICH network, and given the small numbers of $\mathrm{CHs}$ approached, it is not known whether using this method more widely would result in a broad range of $\mathrm{CHs}$ being recruited.

A strength of the study was a rigorous, systematic unbiased method of recruitment, which led to a broad range of $\mathrm{CHs}$ being recruited, as shown in Table 2. This method should also allow for larger scale recruitment in a full-scale trial. However, this method is costly and time-consuming in terms of researcher hours, and may have lengthened the time required to secure $\mathrm{CHs}$ to the study. This systematic method was supplemented by a targeted method of recruitment through a network of research-ready $\mathrm{CHs}$. Requirements for eligibility affected the number of $\mathrm{CHs}$ with research experience, as $\mathrm{REACH}$ eligibility excluded those currently involved in other trials or conflicting studies. Increasing the number of $\mathrm{CHs}$ 
that are familiar with research promotes knowledge and understanding of the processes and benefits for residents and staff.

\section{Conclusions}

A significant amount of time needed to be apportioned for the recruitment of the $\mathrm{CHs}$ to the REACH study. This needs to be taken into account in terms of time and budget when designing similar $\mathrm{CH}$ research, both in terms of the length of engagement required (i.e. a realistic timeline) and the actual hours assigned to researchers for contacting managers to secure and maintain their interest.

Streamlining the processes of recruitment (i.e. simplifying the letter of agreement for $\mathrm{CHs}^{\prime}$ head office approval required to access the $\mathrm{CH}$ ) increased and expedited recruitment. Gaining the approval of larger corporations prior to approaching $\mathrm{CHs}$ may be a method of increasing recruitment and expediting access to the homes. However this must be balanced against a potential for sample bias if the head office purposively select homes. A targeted approach (for example via a network of research-ready $\mathrm{CHs}$ ) may lead to a greater recruitment rate, but due to the small numbers recruited in this study it is not known whether this would lead to a diversity of $\mathrm{CHs}$ being recruited.

\section{Abbreviations}

CH: Care home; CONSORT: Consolidated Standards of Reporting Trials; CQC: Care Quality Commission; CRT: Cluster randomised trial; CTRU: Clinical Trials Research Unit; ENRICH: Enabling Research in Care Homes; NHS: National Health Service; NIHR: National Institute for Health Research; REACH: Research Exploring Physical Activity in Care Homes

\section{Acknowledgements \\ We are pleased to acknowledge the help and support of all the care home staff who participated in the study. We are grateful for the help provided by our research colleagues Gill Procter, Sarah Smith and also Michelle Platton and members of the Yorkshire and the Humber National Institute for Health Research (NIHR) Clinical Research Network. \\ The REACH Programme Team consisted of Karen Birch, School of Biomedical Sciences, University of Leeds, UK; David Ellard, Warwick Clinical Trials Unit, Warwick Medical School, University of Warwick, UK; Amanda Farrin, Leeds Institute of Clinical Trials Research, University of Leeds; Joan Firth, personal and public representative; Anne Forster, Academic Unit of Elderly Care and Rehabilitation, Bradford Institute for Health Research, Bradford Teaching Hospitals NHS Foundation Trust UK and Leeds Institute of Health Sciences, University of Leeds, UK; Bev Gallagher, NHS Bradford Districts Clinical Commissioning Group, UK; Mary Godfrey, Academic Unit of Elderly Care and Rehabilitation, Bradford Institute for Health Research, UK and Leeds Institute of Health Sciences, University of Leeds, UK; Elizabeth Graham, Academic Unit of Elderly Care and Rehabilitation, Bradford Institute for Health Research, Bradford Teaching Hospitals NHS Foundation Trust, UK Claire Hulme, Academic Unit of Health Economics, University of Leeds, UK; Rebecca Lawton, School of Psychology, Faculty of Medicine and Health, University of Leeds, UK; Najma Siddiqi, Department of Health Sciences, Hull York Medical School, University of York, UK; and John Young, Academic Unit of Elderly Care and Rehabilitation, Bradford Institute for Health Research, Bradford Teaching Hospitals NHS Foundation Trust, UK.}

\section{Funding}

This paper presents independent research funded by the NIHR under it Programme Grants for Applied Research (PGfAR) Programme (Grant Reference Number RP-PG-1210-12017). The views expressed in this publication are those of the authors and not necessarily those of the NHS, the NIHR or the Department of Health.

\section{Availability of data and materials}

As this is a feasibility trial to inform a definitive trial, sharing of the trial dataset is not anticipated; however, any data requests should be sent to the corresponding author and would be subject to review by a sub-group of the trial team. All data sharing activities would require a data sharing agreement.

\section{Authors' contributions}

AE led the intervention training and implementation, was responsible for care home recruitment, analysed the data and wrote the manuscript. JA took a lead role in accelerometer data acquisition and analysis, participant recruitment and data collection. RC was responsible for the statistical analysis and reporting of the trial. BC was responsible for the statistical analysis and reporting of the trial. DRE was a grant applicant and is a member of the programme management group. AFa was a grant applicant, is a member of the programme management group, led the statistical and methodological design of the trial and was responsible for the main statistical analysis and reporting. MG took a lead role in intervention development, led intervention training and implementation and was responsible for the implementation and analysis of the process evaluation. $L G$ was responsible for the operational delivery of the feasibility trial at the Clinical Trials Research Unit (CTRU). JG was responsible for the operational delivery of the REACH Programme, analysed the data and wrote the manuscript. VM was responsible for the operational delivery of the trial at the CTRU. NS was a grant applicant and is a member of the programme management group. AFo is the lead grant holder and oversaw the design and implementation of the feasibility trial. All authors were involved in drafting the manuscript or revising it critically for important intellectual content. All authors read and approved the final manuscript.

\section{Ethics approval and consent to participate}

Ethical approval was obtained through the East of England - Cambridgeshire and Hertfordshire Research Ethics Committee (REC) (Ref. 15/EE/0125).

\section{Competing interests}

The authors declare that they have no competing interests.

\section{Publisher's Note}

Springer Nature remains neutral with regard to jurisdictional claims in published maps and institutional affiliations.

\section{Author details}

${ }^{1}$ Academic Unit of Elderly Care and Rehabilitation, Bradford Institute for Health Research, Bradford Teaching Hospitals NHS Foundation Trust, Temple Bank House, Bradford Royal Infirmary, Duckworth Lane, Bradford, West Yorkshire BD9 6RJ, UK. ${ }^{2}$ School of Biomedical Sciences, University of Leeds, Garstang Building, Leeds LS2 9JT, UK. ${ }^{3}$ Clinical Trials Research Unit, Leeds Institute of Clinical Trials Research, University of Leeds, Level 10, Worsley Building, Clarendon Way, Leeds LS2 9NL, UK. ${ }^{4}$ Warwick Clinical Trials Unit, Warwick Medical School, University of Warwick, Coventry CV4 7AL, UK. ${ }^{5}$ Leeds Institute of Health Sciences, University of Leeds, Level 10, Worsley Building, Clarendon Way, Leeds LS2 9NL, UK. ${ }^{6}$ Department of Health Sciences, Hull York Medical School, University of York, ARRC Building, Heslington, York YO10 5DD, UK.

Received: 15 January 2018 Accepted: 12 September 2018

Published online: 03 October 2018

References

1. Population estimates for the UK, England and Wales, Scotland and Northern Ireland: mid 2015. Office for National Statistics; 2015. https://www.ons.gov.uk/ peoplepopulationandcommunity/populationandmigration/populationestimates/ bulletins/annualmidyearpopulationestimates/mid2015\#main-points. Accessed 29 Nov 2017

2. Changes in the older resident care home population between 2001 and 2011. Office for National Statistics; 2014. https://www.ons.gov.uk/ peoplepopulationandcommunity/birthsdeathsandmarriages/ageing/articles/ changesintheolderresidentcarehomepopulationbetween2001and2011/201408-01. Accessed 29 Nov 2017

3. Care Quality Commission The independent regulator of health and social care in England. https://www.cqc.org.uk. Accessed 29 Nov 2017. 
4. Kingston A, Wohland P, Wittenberg R, Robinson L, Brayne C, Matthews FE, et al. Is late life dependency increasing or not? A comparison of the Cognitive Function and Ageing Studies (CFAS). Lancet. 2017;390:P1676-84. https://doi.org/10.1016/S0140-6736(17)31575-1.

5. Gordon AL, Franklin M, Bradshaw L, Logan P, Elliott R, Gladman JRF. Health status of UK care home residents: a cohort study. Age Ageing. 2014:43:97-103

6. Witham MD, George J. Clinical trial design for older people - time for a rethink. Q J Med. 2014;107:15-6.

7. McMurdo MET, Roberts H, Parker S, Wyatt N, May H, Goodman C, et al. Improving recruitment of older people to research through good practice. Age Ageing. 2011;40:659-65.

8. Zermansky AG, Alldred DP, Petty DR, Raynor DK. Striving to recruit: the difficulties of conducting clinical research on elderly care home residents. J R Soc Med. 2007:100:258-61.

9. Sinclair H, Batty JA, Qiu W, Kunadian V. Engaging older patients in cardiovascular research: observational analysis of the ICON-1 study. Open Heart. 2016;3:e000436. https://doi.org/10.1136/openhrt-2016-000436.

10. Wood F, Prout H, Bayer A, Duncan D, Nuttall J, Hood K, et al. Consent, including advanced consent, of older adults to research in care homes: a qualitative study of stakeholders' views in South Wales. Trials. 2013;14:247.

11. Shepherd V, Nuttall J, Hood K, Butler CC. Setting up a clinical trial in care homes: challenges encountered and recommendations for future research practice. BMC Res Notes. 2015;8:306

12. Goodman C, Baron NL, Machen I, Stevenson E, Evans C, Davies SL, et al. Culture, consent, costs and care homes: enabling older people with dementia to participate in research. Aging Ment Health. 2011;15:475-81.

13. Bowman C, Whistler J, Ellerby M. A national census of care home residents. Age Aging. 2004;33:561-6.

14. Davies SL, Goodman C, Manthorpe J, Smith A, Carrick N, lliffe S. Enabling research in care homes: an evaluation of a national network of research ready care homes. BMC Med Res Methodol. 2014;14:47.

15. ENRICH: Enabling Research in Care Homes. http://www.enrich.nihr.ac.uk. Accessed 29 Nov 2017

16. Forster A, Airlie J, Birch K, Cicero R, Cundill B, Ellwood A, et al. Research Exploring Physical Activity in Care Homes (REACH): study protocol for a randomised controlled trial. Trials. 2017:18:182.

17. 2011 Census: Population estimates by five-year age bands, and household estimates, for local authorities in the United Kingdom. Office for National Statistics. https://www.ons.gov.uk/peoplepopulationandcommunity/ populationandmigration/populationestimates/datasets/

2011 censuspopulation estimatesbyfiveyearagebandsandhousehold estimatesforlocalauthoritiesintheunitedkingdom. Accessed 30 Nov 2017.

18. Zermanksy AG, Alldred DP, Petty DR, Raynor DK, Freemantle N, Eastaugh J, et al. Clinical medication review by a pharmacist of elderly people living in care homes — randomised controlled trial. Age Ageing. 2006;35:586-91.

19. Barber SE, Forster A, Birch KM. Levels and patterns of daily physical activity and sedentary behaviour measured objectively in older care home residents in the United Kingdom. J Aging Phys Act. 2015;23:133-43.

20. Whitaker R, Ballard C, Stafford J, Orrell M, Moniz-Cook E, Woods RT, et al. Feasibility study of an optimised person-centred intervention to improve mental health and reduce antipsychotics amongst people with dementia in care homes: study protocol for a randomised controlled trial. Trials. 2013;14:13

21. Hawkins RJ, Prashar A, Lusambili A, Ellard DR, Godfrey M. 'If they don't use it, they lose it': how organisational structures and practices shape residents' physical movement in care home settings. Ageing Soc. 2017:1-26. https:// doi.org/10.1017/S0144686X17000290.

\section{Ready to submit your research? Choose BMC and benefit from:}

- fast, convenient online submission

- thorough peer review by experienced researchers in your field

- rapid publication on acceptance

- support for research data, including large and complex data types

- gold Open Access which fosters wider collaboration and increased citations

- maximum visibility for your research: over $100 \mathrm{M}$ website views per year

At BMC, research is always in progress.

Learn more biomedcentral.com/submissions 